\title{
Metodología para el Dimensionamiento y Optimización de un Concentrador Lineal Fresnel
}

\author{
Fernando Lara ${ }^{(1)}$, Nicolás Velázquez ${ }^{(1)}$, Daniel Sauceda ${ }^{(2)}$ y Alexis Acuña ${ }^{(1)}$. \\ Universidad Autónoma de Baja California, (1) Centro de Estudio de las Energías Renovables del Instituto \\ de Ingeniería, (2) Facultad de Ingeniería, Blvd. Benito Juárez y Calle de la Normal s/n Col. Insurgentes \\ Este, 21280, Mexicali, Baja California-México. (e-mail: lara.fernando@uabc.edu.mx, \\ nicolas.velazquez@uabc.edu.mx, daniel.sauceda@uabc.edu.mx, alexis.acuna@uabc.edu.mx)
}

Recibido May. 08, 2012; Aceptado Jul. 12, 2012; Versión final recibida Ago. 16, 2012

\begin{abstract}
Resumen
Se presenta una metodología de dimensionamiento para concentradores lineales Fresnel de espejos curvos y cavidad invertida tipo concentrador parabólico compuesto. El objetivo es determinar los parámetros geométricos del concentrador que permitan obtener la máxima eficiencia de captación de energía solar. La metodología propuesta utiliza un procedimiento analítico y la técnica de trazado de rayos, por medio de la cual se llevó a cabo un estudio paramétrico evaluando las horas de operación correspondientes a un día representativo del mes crítico de la ciudad de Mexicali Baja California, México. Para un concentrador de siete espejos curvos de $34.3 \mathrm{~cm}$ de ancho y absorbedor tubular de $4.2 \mathrm{~cm}$ de diámetro, las dimensiones resultantes fueron: espaciamiento de $10 \mathrm{~cm}$, distancia focal de $1.8 \mathrm{~m}$ y cavidad de $19.7 \mathrm{~cm}$ de ancho. Con estas dimensiones se obtiene una concentración geométrica de 11.9 y una eficiencia de captación solar de $82.4 \%$.
\end{abstract}

Palabras clave: concentrador lineal de Fresnel, concentrador parabólico, espejos curvos, trazado de rayos.

\section{Methodology for Sizing and Optimization of a Linear Fresnel Reflector}

\begin{abstract}
This paper presents a methodology for the sizing of linear Fresnel reflectors with curved mirrors and inverted compound parabolic concentrator cavity. The objective of the paper is to determine the geometrical parameters of the concentrator to obtain the maximum solar energy collection. The proposed methodology uses an analytical procedure in conjunction with a ray trace technique. A parametrical study evaluating the operation hours for a representative day in the critical month for the city of Mexicali, Baja California, Mexico, was carried out. For a concentrator with seven curved mirrors of $34.3 \mathrm{~cm}$ width and tubular absorber of 4.2 $\mathrm{cm}$ diameter, the resulting dimensions were: $10 \mathrm{~cm}$ spacing between mirrors, $1.8 \mathrm{~m}$ focal distance and 19.7 $\mathrm{cm}$ width cavity. With these dimensions, a geometrical concentration of 11.9 and collection efficiency of $82.4 \%$ are obtained.
\end{abstract}

Keywords: linear Fresnel reflector, parabolic concentrator, curved mirror, ray tracing. 


\section{INTRODUCCIÓN}

La energía solar es una fuente primaria de energía limpia, abundante y renovable que puede ayudar en gran medida a disminuir los problemas relacionados con el cambio climático, al ser utilizada para satisfacer las necesidades de los diferentes sectores de la sociedad. Entre las aplicaciones más comunes se tiene el calentamiento de agua, climatización de espacios, calor de procesos industriales, generación de energía eléctrica, desalación de agua, entre otras. Para llevar a cabo el aprovechamiento del recurso solar en aplicaciones de media $\left(100 \mathrm{a} 400^{\circ} \mathrm{C}\right.$ ) y alta temperatura (mayor a $400^{\circ} \mathrm{C}$ ) es necesario previamente captar, concentrar y convertir la radiación solar en energía térmica mediante un dispositivo de colección solar. En los últimos años han sido desarrollados y probados una gran variedad de colectores solares con concentración para diversas aplicaciones, entre los cuales se encuentra el concentrador lineal Fresnel (CLF), considerado como una de las tecnologías más prometedoras debido a las ventajas que tiene con respecto a otros colectores solares. El CLF tiene la capacidad de satisfacer las necesidades térmicas dentro del rango de los 100 a los $300^{\circ} \mathrm{C}$ (Duffie y Beckman, 1991). Este concentrador se compone de espejos espaciados estratégicamente de manera que la radiación solar que incide en el área de apertura es enviada hacia un absorbedor situado en la zona focal por donde circula un fluido de trabajo.

Actualmente, la técnica de trazado de rayos presenta un renovado interés en el desarrollo de nuevos colectores solares con concentración, permitiendo obtener geometrías más eficientes y económicas. En 1991, Mathur et al. (1991a), desarrollaron una metodología empleando una técnica analítica y otra de trazado de rayos para el diseño de CLF de espejos planos, con el objetivo de evaluar la configuración de tres absorbedores y obtener la concentración local; en dicho trabajo se realizó un estudio comparativo entre la metodología analítica y la de trazado de rayos concluyendo que esta última permitía obtener mejores resultados. En ese mismo año, Mathur et al. (1991b), reportaron un estudio relacionado con el descrito anteriormente, donde se analiza el comportamiento óptico-geométrico de un CLF con diferente ancho de espejos. El método empleado fue el mismo que el utilizado en Mathur et al. (1991a), y se basó en la obtención del valor apropiado del espaciamiento horizontal entre espejos, a partir del sombreado y el bloqueo nulo de los rayos reflejados en la máxima altura solar.

Algunos autores, tales como Sootha y Negi (1994), Singh et al. $(1999,2010)$ y Velázquez et al. (2010) estudiaron el diseño de CLF para diversas aplicaciones utilizando el método propuesto por Mathur et al. (1990, 1991a, 1991b). En el 2000, Mills y Morrison (2000), con ayuda de un modelo de trazado de rayos generaron los mapas de colección de un nuevo concepto de colector llamado "concentrador lineal de Fresnel compacto"; este diseño permite maximizar el área de cobertura del concentrador obteniendo arreglos más densos y libres de sombreados o bloqueos entre espejos. En el 2006, Häberle et al. (2006), desarrollaron un CLF con cavidad receptora tipo Concentrador Parabólico Compuesto (CPC) y tubo absorbedor al vacío. En dicho estudio se optimizó el diseño geométrico del colector y se obtuvo la eficiencia óptica del prototipo mediante un software de trazado de rayos, sin embargo no se presentó una metodología para el dimensionamiento de futuros concentradores.

En el 2010, otro prototipo de CLF a construir fue optimizado geométricamente por Barale et al. (2010); el estudio se centró en la optimización de los espejos y en el mecanismo de seguimiento, aunque también se evaluaron diversas propuestas de cavidades receptoras, incluyendo geometrías tipo CPC con la variante de receptor con aire y al vacío. Como resultados se logró obtener la eficiencia óptica del prototipo a partir de una herramienta propia de diseño, además de evaluar la influencia de la variación de los parámetros y la energía captada. En el 2011, Grena y Tarquini (2011), propusieron una configuración de CLF que incorpora un diseño de cavidad compuesto por dos alas parabólicas abiertas. El estudio logró determinar las eficiencias ópticas del sistema mediante un análisis de trazado de rayos computarizado en C++.

Durante ese mismo año, otro método para optimizar el espaciamiento entre espejos se presentó buscando maximizar la exergía del concentrador, las horas operacionales y minimizar los costos (Nixon y Davies, 2011). El software Optica ${ }^{\circledR}$ de trazado de rayos fue el utilizado para evaluar la eficiencia óptica del concentrador. Los resultados obtenidos fueron favorables y se espera obtener mejoras significativas en una gran variedad de aplicaciones.

Dado que en los estudios previos sobre el dimensionamiento de CLF con espejos curvos no se ha planteado una metodología clara y sencilla para el diseño de sus componentes, en este trabajo se presenta una metodología híbrida entre un procedimiento analítico (modelado geométrico) y la técnica de trazado de rayos utilizando el programa Trace Pro $R C^{\circledR}$, que permite dimensionar y evaluar la influencia de los diferentes parámetros geométricos sobre la energía captada por la cavidad del CLF, considerando el día representativo del mes crítico, el número de horas de operación, las pérdidas en los extremos, tamaño de la imagen de los rayos concentrados, sombreado de cavidad y estructura y bloqueo entre espejos, entre otras. 


\section{DESCRIPCIÓN DEL SISTEMA}

Con base en que los CLF pueden tener múltiples aplicaciones, se propone un diseño bajo el concepto modular por medio del cual, desarrollando arreglos en serie o en paralelo de $4 \mathrm{~m}$ de longitud $\left(L_{C L F}\right)$ podemos satisfacer las diferentes necesidades tanto en nivel de temperatura como cantidad de energía. En la Fig. 1 se muestra esquemáticamente el sistema en estudio, el cual consta de una estructura, siete espejos curvos (tres espejos por lado y un espejo central), un mecanismo de seguimiento, una cavidad tipo CPC con cubierta de vidrio y absorbedor tubular y un circuito de tuberías. El sistema de seguimiento es de tipo persiana pudiendo utilizar sensores o un sistema de ecuaciones para encontrar la posición del Sol.

El principio operativo del CLF consiste en captar y redireccionar hacia la cavidad la radiación solar incidente sobre el área de apertura de los espejos curvos. Una fracción de la energía concentrada que atraviesa la cubierta de vidrio de la cavidad es redireccionada por el concentrador secundario tipo CPC, el cual se encarga de hacerla incidir sobre el absorbedor tubular donde es atrapada por una superficie selectiva de alta absorbancia. La energía solar atrapada por el absorbedor se transmite a un fluido caloportador que circula por la parte interna del tubo, obteniéndose como calor útil, el cual será aprovechado para el suministro de energía térmica a un sistema de aire acondicionado solar.

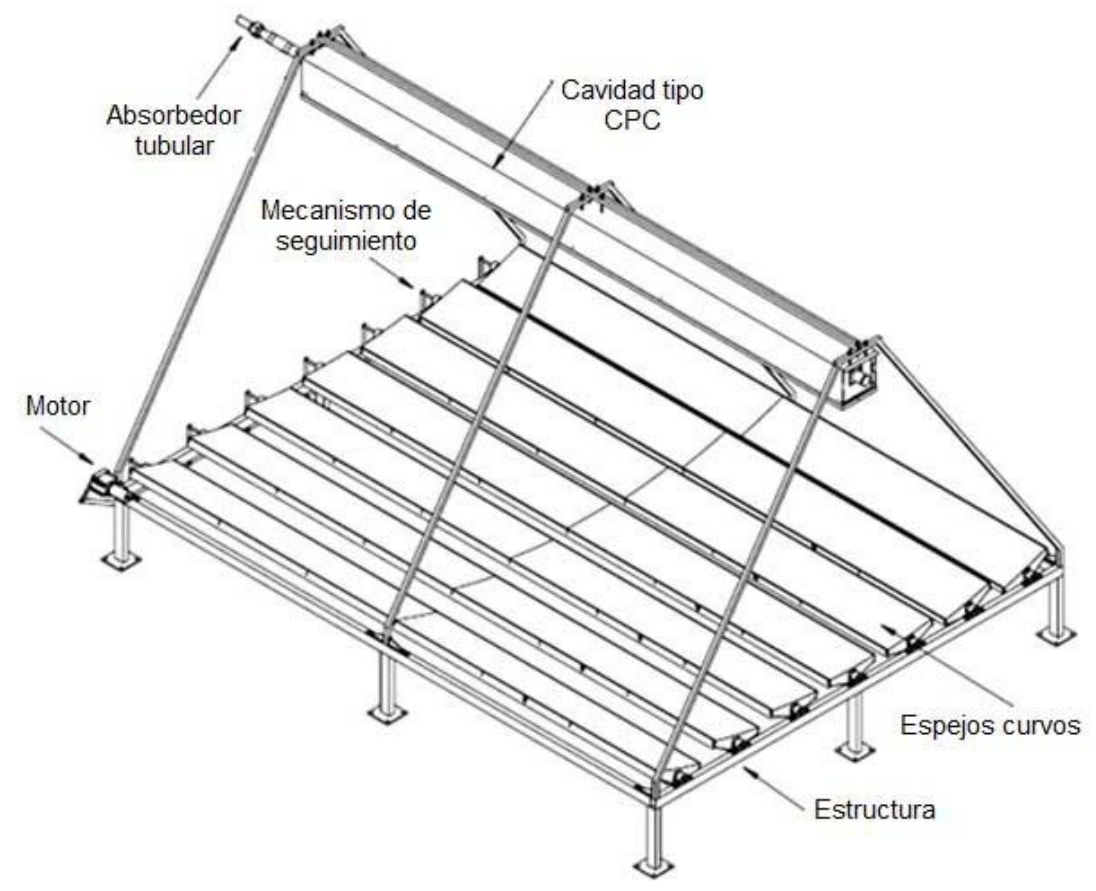

Fig. 1: Concentrador lineal Fresnel de espejos curvos y cavidad CPC

\section{DISEÑO DEL CONCENTRADOR}

El diseño del CLF está planteado para llevarse a cabo en dos etapas. La primera corresponde al análisis geométrico de los espejos curvos y de la cavidad tipo CPC, mientras que la segunda describe la metodología de dimensionamiento y optimización del CLF completo.

\section{Análisis geométrico}

En la Fig. 2 se muestran los parámetros geométricos característicos de los espejos curvos, apoyándonos en la geometría de la parábola y en su comportamiento al interactuar con la radiación solar se establecen en la Tabla 1 las ecuaciones que serán utilizadas en los proceso iterativos planteados en la metodología de dimensionamiento y optimización. 

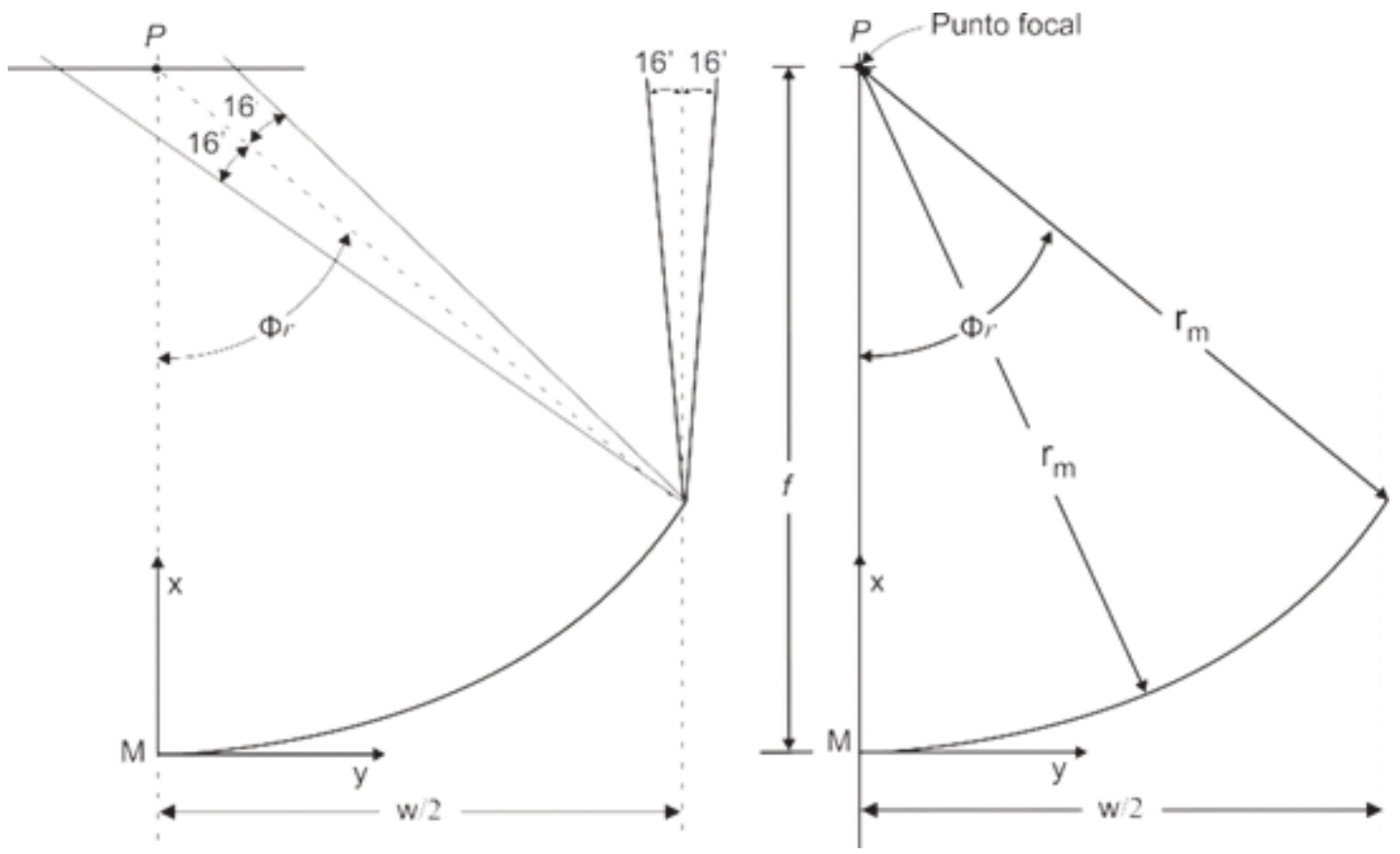

Fig. 2: Parámetros geométricos de los espejos curvos

Tabla 1: Ecuaciones correspondientes a los parámetros geométricos de los espejos del CLF

\begin{tabular}{|l|c|c|}
\hline $\begin{array}{l}\text { Ecuación de la parábola. Duffie y Beckman } \\
(1991)\end{array}$ & $y^{2}=4 f x$ & (1) \\
\hline Ángulo de apertura. Duffie y Beckman (1991) & $\varphi_{r}=\tan ^{-1}\left[\left(8\left[\frac{f}{w}\right]\right) /\left(16\left[\frac{f}{w}\right]^{2}-1\right)\right]$ & (2) \\
\hline Profundidad del espejo. & $\mathrm{p}=f-\left[\left(\left(\frac{w}{2}\right) /\left(\tan \left(\phi_{r}\right)\right)\right)\right]$ & (3) \\
\hline
\end{tabular}

En la Fig. 3 se muestra el esquema de la cavidad tipo CPC, la cual está compuesta por una sección simétrica de parábolas e involutas y por un absorbedor tubular de diámetro $\left(D_{\mathrm{a}}\right)$. El ancho de cavidad $\left(W_{\text {cav }}\right)$ corresponde al tamaño de la imagen de los rayos concentrados por los espejos curvos considerando el sol en la hora crítica, teniendo en cuenta que dicha hora es establecida dependiendo de las horas de captación requeridas y dando especial atención al último espejo curvo que es donde se tiene la mayor dispersión de energía.

En cuanto a (Ca ), se tiene que es el ángulo que define la máxima concentración alcanzada por la cavidad y se obtiene a partir del eje vertical de la cavidad CPC con respecto al último espejo curvo del CLF, considerando la trayectoria del rayo a través del borde de la parábola $(O)$ y la tangente al tubo absorbedor (T). En la Tabla 2, se encuentran enlistadas las ecuaciones utilizadas para el diseño de la cavidad.

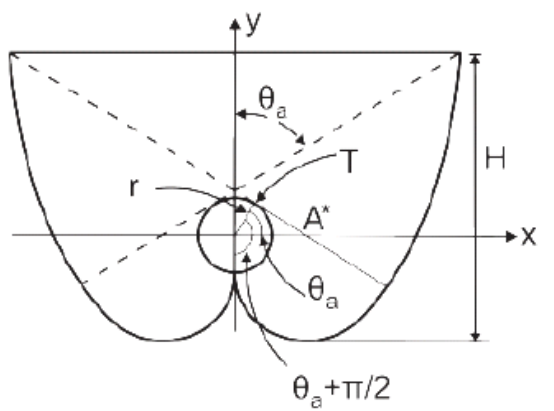

Sección de parábola

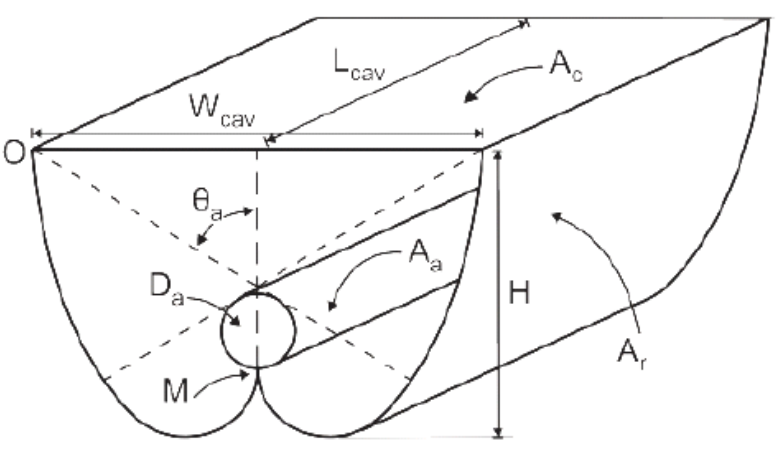

Sección de parábola e involuta

Fig. 3: Esquema de la cavidad tipo CPC con absorbedor tubular 
Tabla 2: Ecuaciones geométricas para la cavidad CPC

\begin{tabular}{|c|c|c|c|}
\hline \multicolumn{2}{|c|}{$\begin{array}{c}\text { Concentración geométrica del CPC. Duffie y } \\
\text { Beckman (1991) }\end{array}$} & $C_{C P C}=\frac{1}{\operatorname{sen}\left(\theta_{a}\right)}$ & $(4)$ \\
\hline \multirow{3}{*}{ 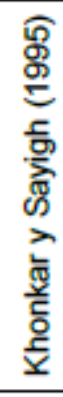 } & $\begin{array}{c}\text { Ecuaciones para establecer la } \\
\text { sección parabólica en el plano }(x, y)\end{array}$ & $\begin{array}{c}x=r\left(\operatorname{sen} \phi-A^{*} \cos \phi\right) \\
y=-r\left(A^{*} \operatorname{sen} \phi+\cos \phi\right), \\
\frac{\pi}{2}+\theta_{a} \leq \phi \leq \frac{3 \pi}{2}-\theta_{a}\end{array}$ & $\begin{array}{l}(5) \\
(6)\end{array}$ \\
\hline & $\begin{array}{l}\text { Distancia formada desde la tangente } \\
\text { del absorbedor al inicio de la parábola }\end{array}$ & $\begin{array}{c}A^{*}=\frac{\frac{\pi}{2}+\theta_{a}+\phi-\cos \left(\phi-\theta_{a}\right)}{1+\operatorname{sen}\left(\phi-\theta_{a}\right)} \\
\text { Donde para este punto: } \phi=\theta_{a}+\frac{\pi}{2}\end{array}$ & $(7)$ \\
\hline & $\begin{array}{c}\text { Ecuaciones para establecer la } \\
\text { sección de involuta en el plano }(x, y)\end{array}$ & $\begin{array}{c}x=r(\operatorname{sen} \phi-\phi \cos \phi) \\
y=r(\phi \operatorname{sen} \phi+\cos \phi), \quad 0 \leq \phi \leq \frac{\pi}{2}+\theta_{a}\end{array}$ & $\begin{array}{l}(8) \\
(9)\end{array}$ \\
\hline & Área del reflector CPC & $A_{r}=\sum_{i=0}^{\frac{5}{2} \pi} \sqrt{\left(x^{2}+y^{2}\right)}$ & $(10)$ \\
\hline & Área del absorbedor tubular & $A_{a}=\pi D_{a} L_{C L F}$ & $(11)$ \\
\hline & Concentración geométrica del CLF & $C_{C L F}=\sum_{i=1}^{N} \frac{w \cos \left(\theta_{i}\right)}{W_{c a v}}$ & $(12)$ \\
\hline
\end{tabular}

\section{Metodología de dimensionamiento y optimización del CLF}

En forma general la metodología que se presenta en la Fig. 4 consiste en un procedimiento iterativo exploratorio que por medio de las ecuaciones planteadas se propone una configuración geométrica y se aplica la técnica de trazado de rayos (programa Trace Pro $R C^{\mathbb{B}}$ ) para realizar el dimensionamiento completo y una evaluación técnica del CLF, considerando el día representativo del mes crítico, el número de horas de operación requeridas, pérdidas en los extremos, tamaño de la imagen de los rayos concentrados, sombreado de cavidad, bloqueo entre espejos, entre otras.

Existen algunos aspectos importantes dentro del algoritmo metodológico que son necesarios en las configuraciones geométricas evaluadas de CLF. Debido a que existe una relación de giro entre los espejos reflectores (efecto persiana), para cada configuración en análisis se realiza un trazado de rayos que nos permite establecer la inclinación de cada uno de los espejos y el mecanismo de seguimiento, de tal forma que se asegure la captación del recurso solar por la cavidad en las diferentes horas del día. Otro aspecto importante a considerar es el ancho máximo de apertura de la cavidad, el cual se obtiene enfocando el sistema de seguimiento del colector en la hora crítica del día a evaluar (imagen más grande en el punto focal), de tal forma que toda la energía redireccionada por los espejos curvos sea captada por el concentrador secundario tipo CPC. Para establecer el espaciamiento entre espejos $\left(S_{n}\right)$ se hace una exploración desde cero separación hasta tener cero sombreados entre ellos, seleccionando la separación que nos proporcione la máxima captación de radiación solar en el día crítico. Hay que considerar que existen situaciones en las cuales el espacio disponible resulta ser restrictivo, por lo que es necesario sacrificar el espaciamiento adecuado del diseño sin afectar de manera considerable la captación de radiación solar. Finalmente, en forma general se selecciona la configuración geométrica que nos proporcione la máxima eficiencia de captación solar con el mínimo de requerimiento de espacio.

La metodología propuesta contiene las siguientes consideraciones: el concentrador tiene un sistema de seguimiento ideal (se mueve a la misma velocidad aparente del Sol), los espejos tienen una reflectancia definida constante y tienen una reflexión especular, pudiendo modificarse a cualquier tipo de propiedades ópticas; la cubierta de la cavidad de tipo CPC tiene una transparencia definida constante, que puede variar dependiendo el caso en estudio, el trazado de rayos se hace sin considerar el ángulo subtendido del Sol, también se establece que cada rayo trazado contiene un Watt de energía y los espejos curvos mantienen el mismo ancho (w). 


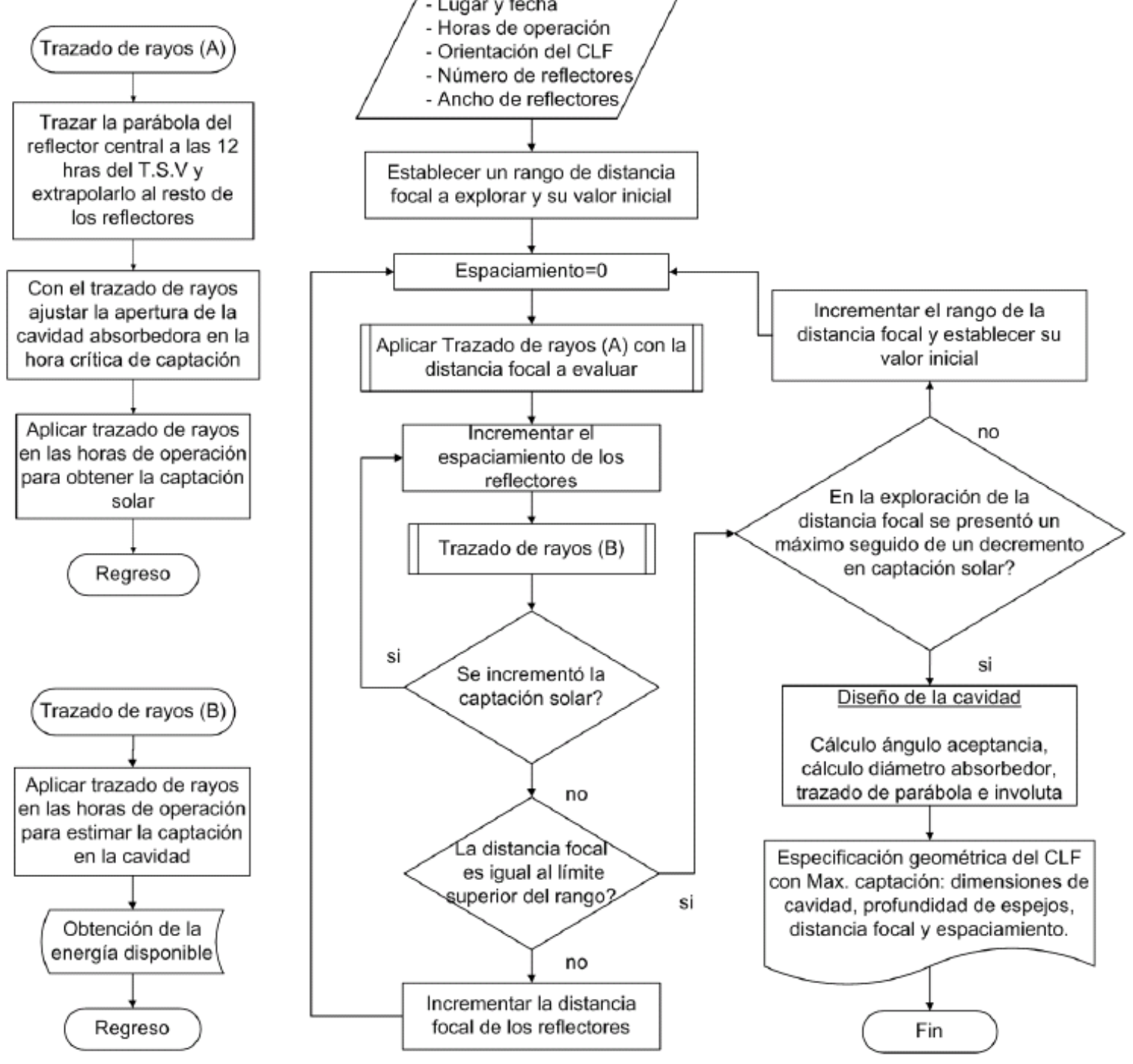

Fig. 4: Algoritmo metodológico para el dimensionamiento y optimización del CLF

\section{RESULTADOS}

El estudio se realizó para la ciudad de Mexicali B.C, México, (latitud: $32^{\circ} 39^{\prime} 26.2^{\prime \prime} \mathrm{N}$ y longitud: $115^{\circ} 27^{\prime}$ 3.58" O) considerando que el concentrador será utilizado para satisfacer las necesidades energéticas de una unidad de enfriamiento solar por absorción. Para esta aplicación se tiene a agosto como mes crítico (máxima demanda de energía) y se utiliza el día 16 como representativo del mes, de acuerdo con Duffie y Beckman (1991). Buscando obtener la mayor cantidad de energía, el CLF se posicionó con una orientación norte-sur con seguimiento este-oeste (Lentz et al., 2009). El estudio operativo se realizó de las 8:00 AM a 4:00 PM en tiempo solar verdadero (TSV). En este caso se consideraron 7 espejos de $34.30 \mathrm{~cm}$ de ancho y 4 metros de largo debido a que se pretende manejar el concepto modular con el mínimo de desperdicios de material (adaptándonos a los materiales existentes en el mercado).

En la Tabla 3 se muestran los parámetros utilizados en cada hora a evaluar. Los valores correspondientes a la radiación solar directa en la superficie horizontal $\left(I_{b}\right)$ han sido utilizados para establecer la retícula de rayos que simula la radiación solar incidente sobre el plano. En cuanto a la ubicación de la fuente de energía (Sol) se han utilizado los valores correspondientes a los ángulos de altura solar $\left(h_{s}\right)$ y acimutal solar $\left(g_{s}\right)$. 
Tabla 3: Parámetros de entrada del programa de trazado de rayos para el 16 de agosto

\begin{tabular}{|l|l|c|c|c|}
\hline Hora TSV & Hora TC & Altura solar ${ }^{\circ}\left(\mathrm{h}_{\mathrm{s}}\right)$ & Acimutal solar ${ }^{\circ}\left(\mathrm{g}_{\mathrm{s}}\right)$ & $\mathrm{I}_{b} \mathrm{~W} / \mathrm{m}^{2}$ \\
\hline 8:00 AM & 8:47 AM & 32.46 & -85.41 & 245.8 \\
\hline 9:00 AM & 9:47 AM & 44.91 & -75.62 & 364.72 \\
\hline 10:00 AM & 10:47 AM & 56.69 & -61.86 & 469.34 \\
\hline 11:00 AM & 11:47 AM & 66.51 & -38.72 & 541.04 \\
\hline 12:00 PM & 12:47 PM & 70.82 & 0 & 566.54 \\
\hline
\end{tabular}

En la Fig. 5, se muestran los resultados del comportamiento horario para una configuración geométrica con espaciamiento nulo entre espejos. En dicha figura se puede observar como en las primeras horas de captación, el bloqueo de energía (rayos solares) ocasionado por los espejos afecta de manera considerable la concentración de radiación solar. A medida que las horas transcurren y nos acercamos a la máxima altura solar (12:00 PM del TSV) el bloqueo se ve reducido al presentarse una menor inclinación de los espejos, la cual también produce un incremento en el área efectiva de captación, provocando un aumento en la captación de radiación solar.

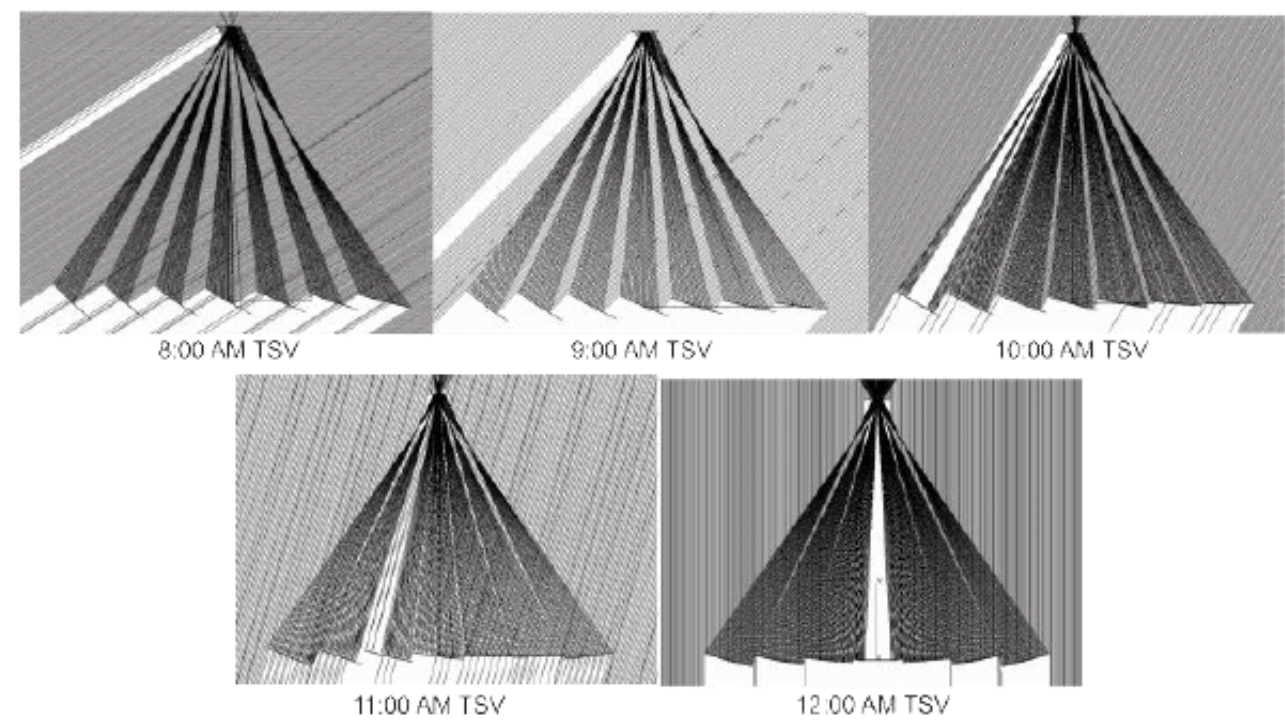

Fig. 5: Comportamiento horario del CLF con distancia focal de $1.8 \mathrm{~m}$ y espaciamiento nulo

Posteriormente, por la tarde o caída del Sol se tiene que el fenómeno ocurre de manera contraria, obteniéndose la máxima captación solar a las 12:00 PM con el mínimo tamaño de imagen de los rayos reflejados en el punto focal. Por lo anterior el CLF proporciona una cantidad y calidad de la energía variable durante el día, lo cual debe de tomarse en cuenta en los diseños de los sistemas de control de las diferentes aplicaciones, para que no sea una desventaja con respecto a otras fuentes de energía sin esta variabilidad o intermitencia.

En la Fig. 6 se muestra una configuración geométrica similar a la establecida en la Fig. 5, con la diferencia de que el parámetro en evaluación corresponde a un espaciamiento entre espejos de $10 \mathrm{~cm}$. Es necesario mencionar que esta geometría mantiene el ancho de cavidad de la configuración geométrica anterior (Fig. 5), la cual se obtuvo captando la radiación solar concentrada del campo de reflexión en el ancho de cavidad $\left(W_{\text {cav }}\right)$ empleando un espaciamiento nulo en la hora crítica de operación (8:00 AM del TSV), generando un ancho máximo que se ha fijado para las configuraciones con espaciamientos de 10 y $20 \mathrm{~cm}$. Al comparar la Fig. 6 con respecto a la Fig. 5, se tiene que en las primeras horas del día la captación de radiación solar se ve beneficiada debido a que se tiene un menor bloqueo entre espejos, sin embargo, conforme el espaciamiento se incrementa, la inclinación de los espejos también aumenta provocando un ligero desaprovechamiento del área de captación de los espejos. Es importante mencionar que el espaciamiento entre espejos deberá ser seleccionado considerando también la dispersión de los rayos solares en el trayecto del espejo hacia la cavidad, tomando en cuenta que conforme se aumenta dicho espaciamiento mayor dispersión de energía se tiene, aunado con un incremento en las dimensiones del campo de reflexión. 

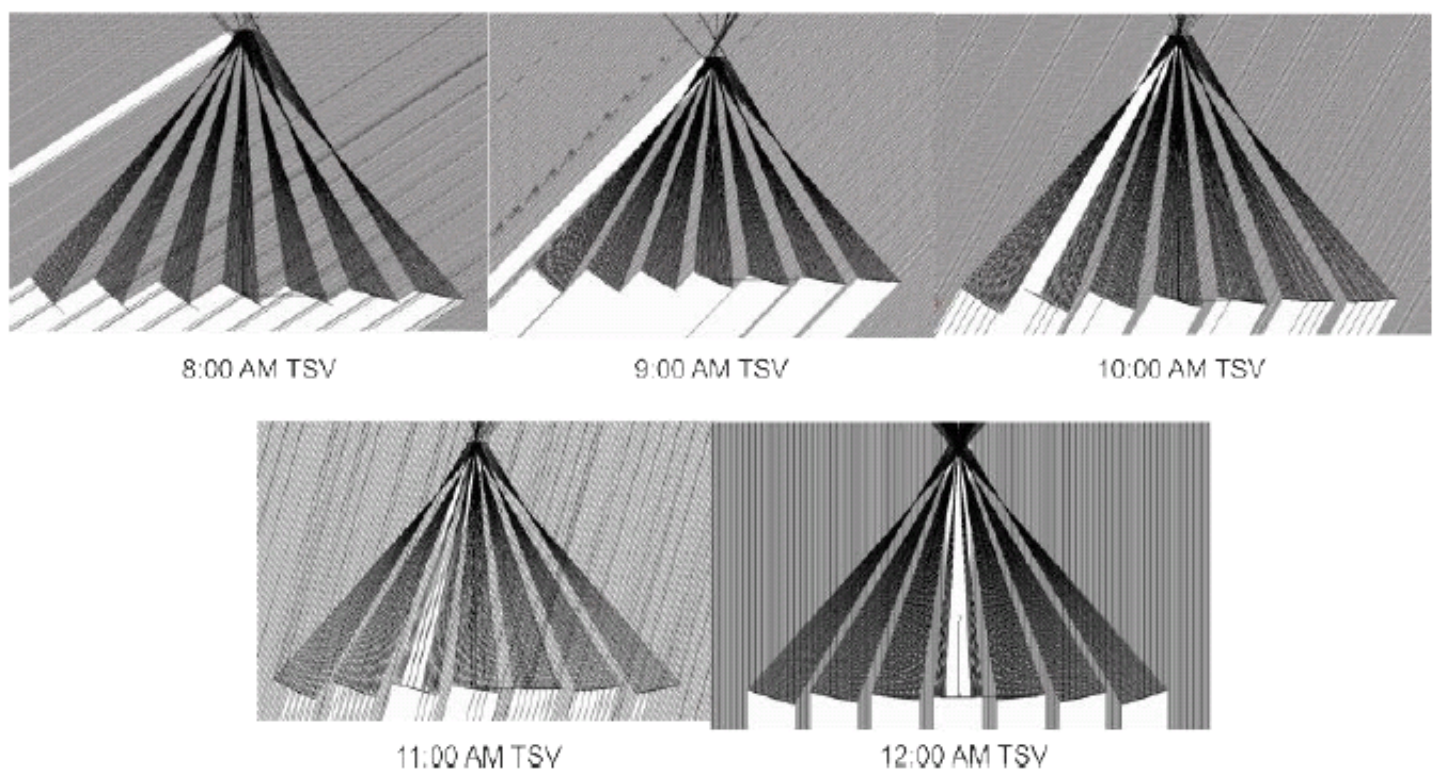

Fig. 6: Comportamiento horario del CLF con distancia focal de $1.8 \mathrm{~m}$ y espaciamiento de $10 \mathrm{~cm}$

Otro parámetro que tiene una fuerte influencia en la captación de la radiación solar es la distancia focal del concentrador, ya que afecta el tamaño de la imagen de los rayos solares concentrados (ancho de cavidad requerido), las pérdidas de energía en los extremos, el sombreado entre espejos y el área efectiva de estos. El procedimiento llevado a cabo para la obtención de la distancia focal y el tamaño de imagen óptimo, consistió primeramente en establecer un rango de distancias focales a evaluar, posteriormente para cada uno de los casos se trazó la parábola del reflector central a las 12:00 PM del TSV, la cual se extrapoló hacia al resto de los reflectores de la configuración en estudio. Una vez establecida la profundidad de la parábola y el ancho de la cavidad (tamaño de imagen mínima) se procedió a estimar la captación en la cavidad durante las horas de operación bajo diversos espaciamientos entre espejos, con la finalidad de obtener la geometría con la máxima eficiencia de captación solar. El estudio paramétrico efectuado mostró que para un CLF de distancia focal pequeña, el ancho de la cavidad resultó ser mayor que el obtenido en distancias focales superiores, debido a que la inclinación y la profundidad de los espejos curvos son más pronunciados, lo que ocasiona que se produzca una mayor dispersión de energía en el punto focal (ver Fig. 7a). Conforme la distancia focal se incrementa, las horas con sombreado disminuyen, requiriendo menor espaciamiento entre espejos (ver Fig. 7b), sin embargo se produce un incremento en las pérdidas de energía en los extremos del concentrador; cabe mencionar que este último factor se ve mayormente afectado cuando se emplean ángulos de incidencia pronunciados que provocan que la radiación solar redireccionada por los espejos no logre incidir en los extremos de la cavidad (ver Fig. 8).
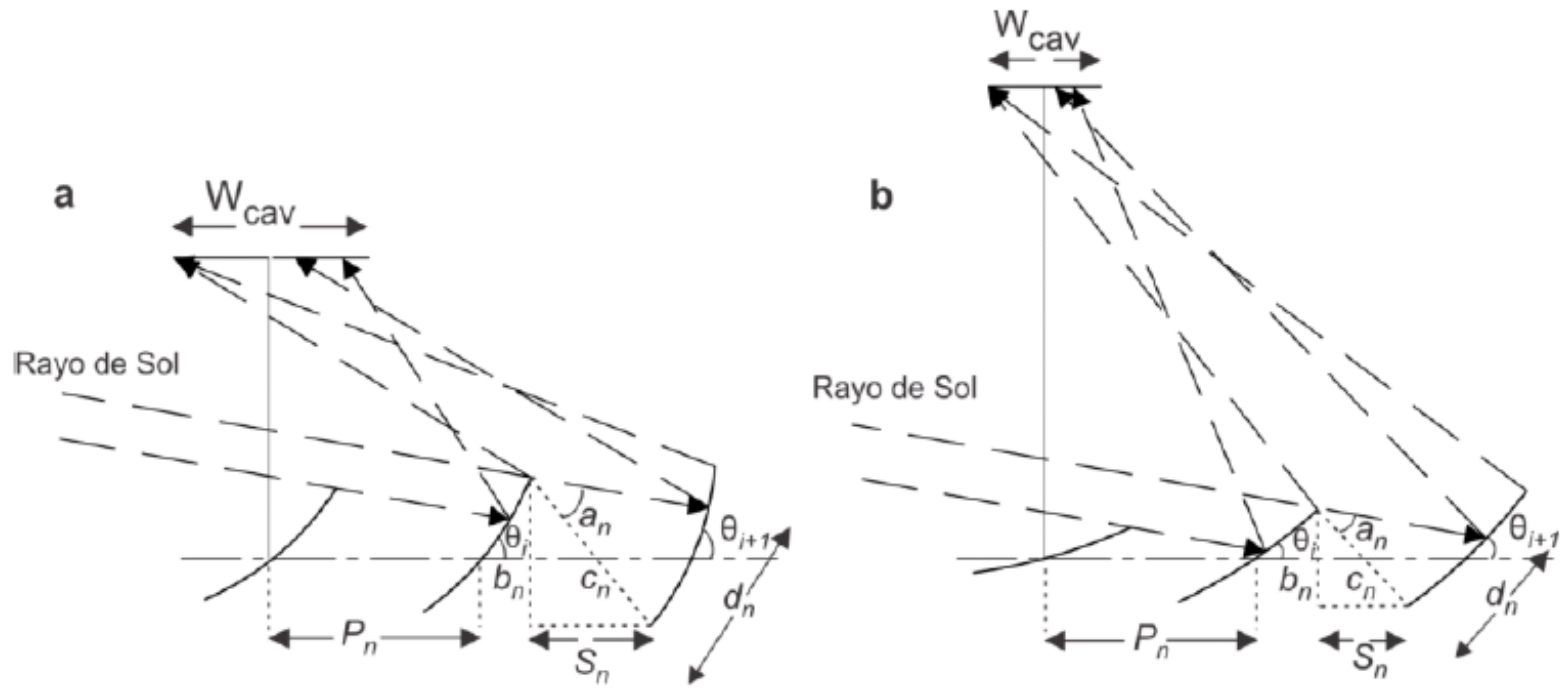

Fig. 7: Configuraciones de geometrías de CLF bajo diversas distancias focales. (a) distancia focal mínima, ancho de cavidad máxima, (b) distancia focal máxima, ancho de cavidad mínima 


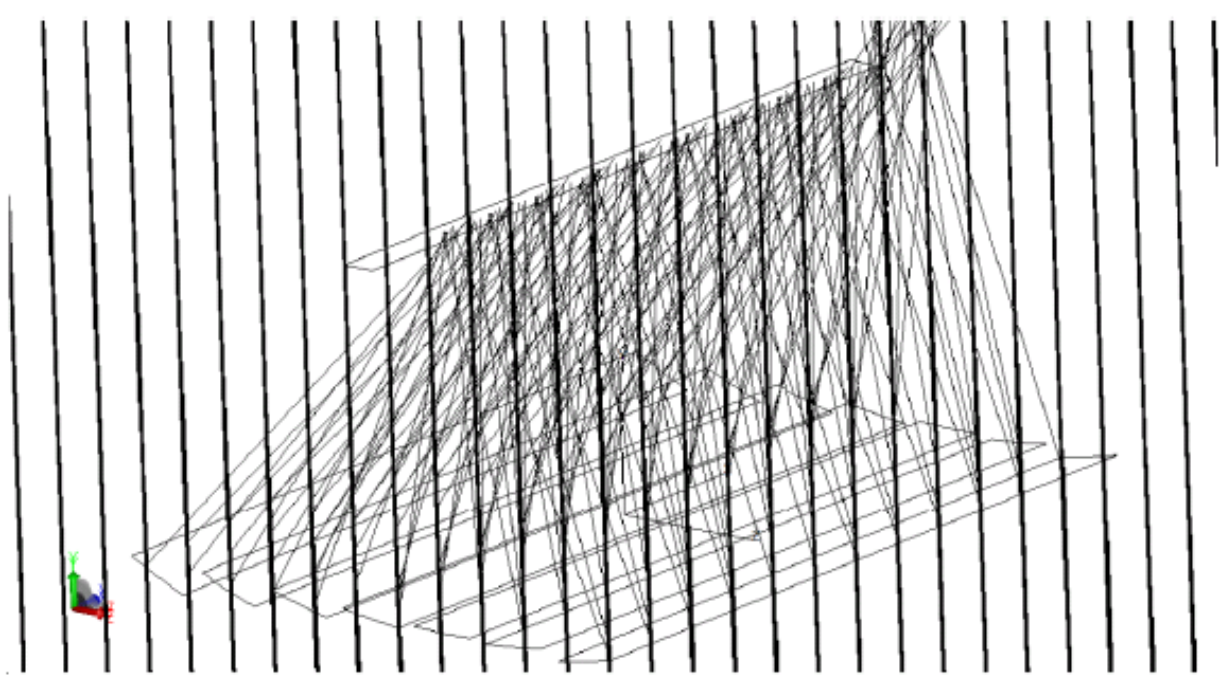

Fig. 8: Pérdidas de energía en los extremos

Con base en lo mencionado y en los resultados obtenidos, el diseño óptimo se estableció a partir del compromiso generado entre el número de horas de operación, las pérdidas en los extremos, tamaño de la imagen de los rayos concentrados, sombreado de cavidad y el bloqueo entre espejos.

En las Fig. 9, 10, 11 y 12 se muestran los resultados obtenidos en el estudio de las diferentes configuraciones geométricas de los CLF evaluados, las cuales son definidas estableciendo una distancia focal con tamaño de imagen mínima y realizando un barrido a diversos espaciamientos. Si analizamos los resultados de la exploración con diferentes espaciamiento entre los espejos en cada una de las distancias focales evaluadas, podemos apreciar que el espaciamiento de $10 \mathrm{~cm}$ resultó ser mejor en las cuatro gráficas mostradas; en la Fig. 9 se tienen tres configuraciones geométricas de CLF con una distancia focal de $1.6 \mathrm{~m}$ y espaciamientos entre espejos de 0,10 y $20 \mathrm{~cm}$; los resultados obtenidos muestran que para la separación de $10 \mathrm{~cm}$ se alcanzó una captación de $3.90 \mathrm{~kW}$ a las 12:00 PM, logrando un compromiso entre el bloqueo de espejos, el área efectiva de captación y el sombreado de la cavidad en el campo de reflexión, principalmente. Seguido a esto, se tuvo que para el espaciamiento de $20 \mathrm{~cm}$ también se produjo una reducción en dichos bloqueos de espejos, sin embargo generó un desaprovechamiento del área efectiva de captación causada por el espaciamiento y por la inclinación de los espejos. En cuanto a la separación de 0 $\mathrm{cm}$, se tuvo que se generó un mayor número de bloqueos ocurridos entre los espejos durante las primeras y últimas horas de operación, ya que la cercanía entre estos afectó considerablemente la captación, dando como resultado la configuración con la captación más baja de energía.

En el caso de las configuraciones geométricas mostradas en las Fig. 10 y 12, se produjo el mismo comportamiento obtenido en la Fig. 9, estableciendo que las configuraciones con mayor captación en ambos casos corresponden a la distancia focal de 1.8 y $2.2 \mathrm{~m}$ con espaciamiento de $10 \mathrm{~cm}$, las cuales resultaron prácticamente con la misma captación de 3.82 kW.

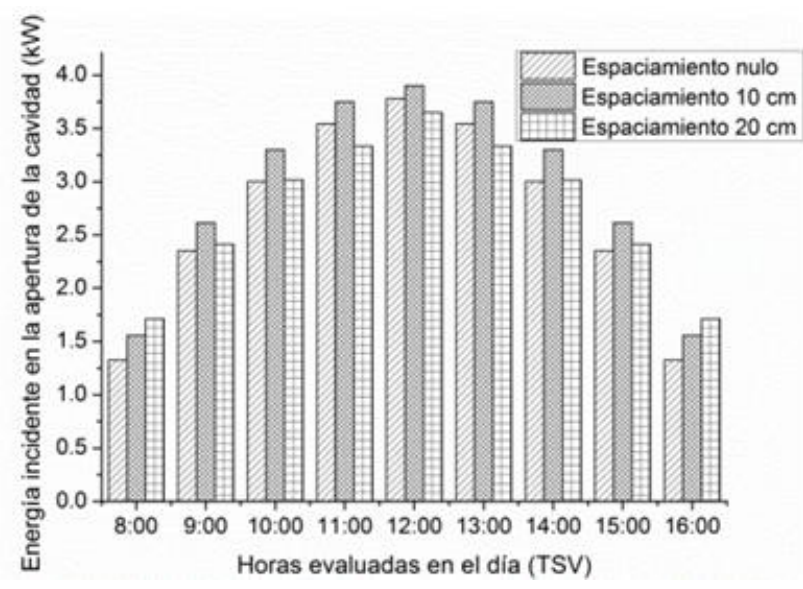

Fig. 9: Evaluación de la distancia focal de $1.6 \mathrm{~m}$ a diversos espaciamientos con un ancho de cavidad de $19.60 \mathrm{~cm}$

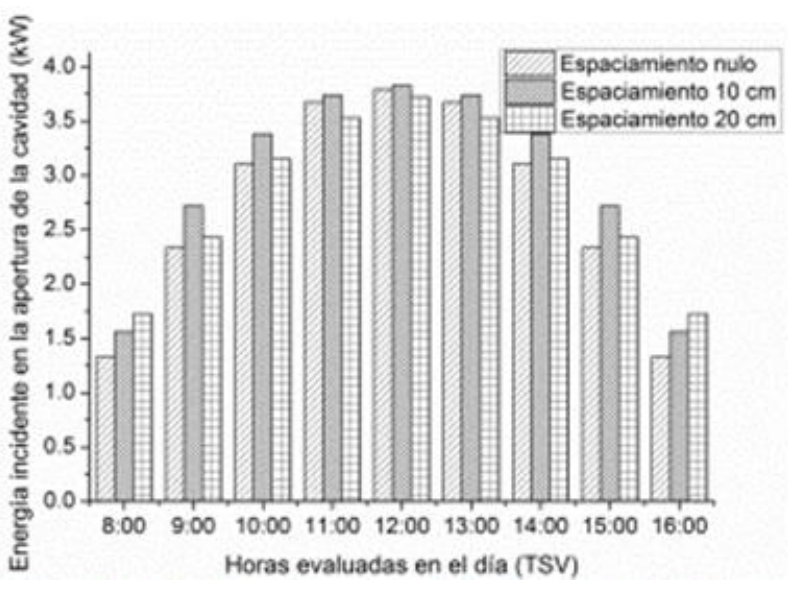

Fig. 10: Evaluación de la distancia focal de $1.8 \mathrm{~m}$ a diversos espaciamientos con un ancho de cavidad de $17.45 \mathrm{~cm}$ 


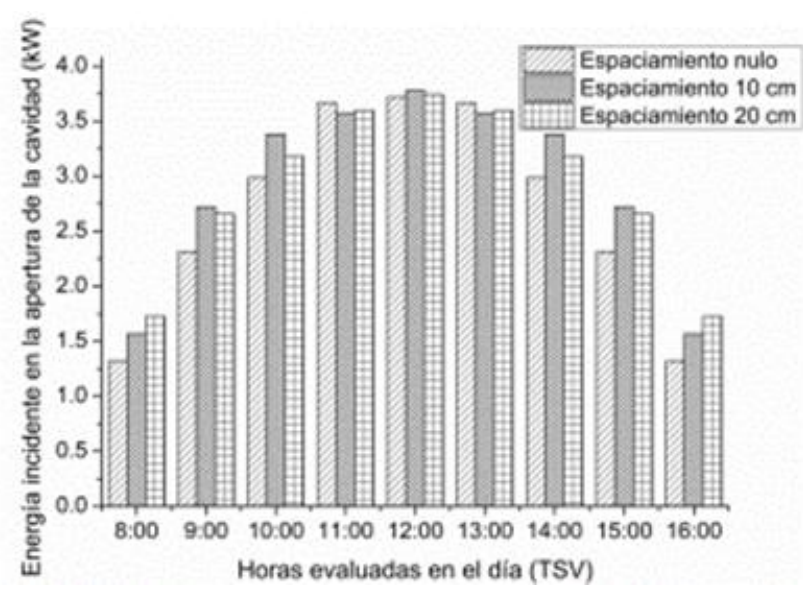

Fig. 11: Evaluación de la distancia focal de $2 \mathrm{~m}$ a diversos espaciamientos con un ancho de cavidad de $15.50 \mathrm{~cm}$

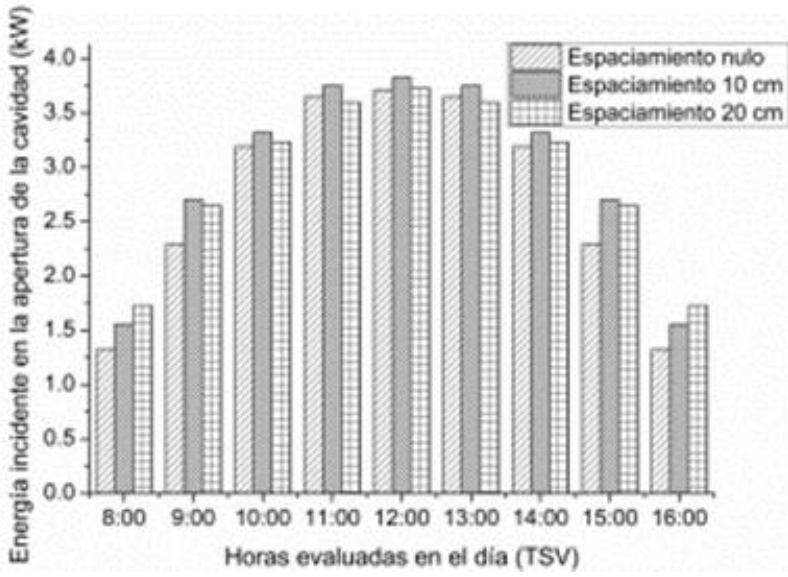

Fig. 12: Evaluación de la distancia focal de 2.2 $\mathrm{m}$ a diversos espaciamientos con un ancho de cavidad de $13.30 \mathrm{~cm}$

Si analizamos el comportamiento obtenido en la Fig. 11 con respecto a las Fig. 9, 10 y 12, vemos que en la mayoría de las horas evaluadas ocurre un fenómeno similar, a diferencia de las 11:00 AM del espaciamiento de $10 \mathrm{~cm}$ donde se generó un sombreado total por la cavidad en el área de apertura de un espejo, provocando una disminución en la captación de radiación solar en dicha hora. También se puede ver que para ese mismo espaciamiento la máxima captación correspondiente es de 3.77 kW, y concierne a la captación más baja de las cuatro distancias focales evaluadas.

Con la finalidad de seleccionar una configuración geométrica óptima de CLF se promedió la captación solar del día para las diversas configuraciones evaluadas buscando la máxima captación diaria (ver Fig. 13). Analizando el promedio para cada caso, se obtuvo que la captación máxima pertenece a la geometría de distancia focal de $1.8 \mathrm{~m}$ con espaciamiento de $10 \mathrm{~cm}$ con una captación de $2.96 \mathrm{~kW}$, seguido de la geometría de distancia focal de $2.2 \mathrm{~m}$ con espaciamiento de $10 \mathrm{~cm}$ y una captación de $2.94 \mathrm{~kW}$. Si observamos el beneficio generado por el espaciamiento en las dos geometrías se puede apreciar que para el primer caso se logró incrementar la captación en un $7.37 \%$ y para el segundo caso un $7.01 \%$, sin embargo, debido a que la captación existente entre ambas configuraciones no presenta una diferencia significativa, se optó por seleccionar la geometría de distancia focal de $1.8 \mathrm{~m}$, tomando como base que dicha geometría presenta una menor complejidad en la fabricación de la cavidad (colocación de la lámina reflectiva en la cavidad) y a su vez un menor costo de fabricación de la estructura para la colocación de la cavidad en el punto focal.

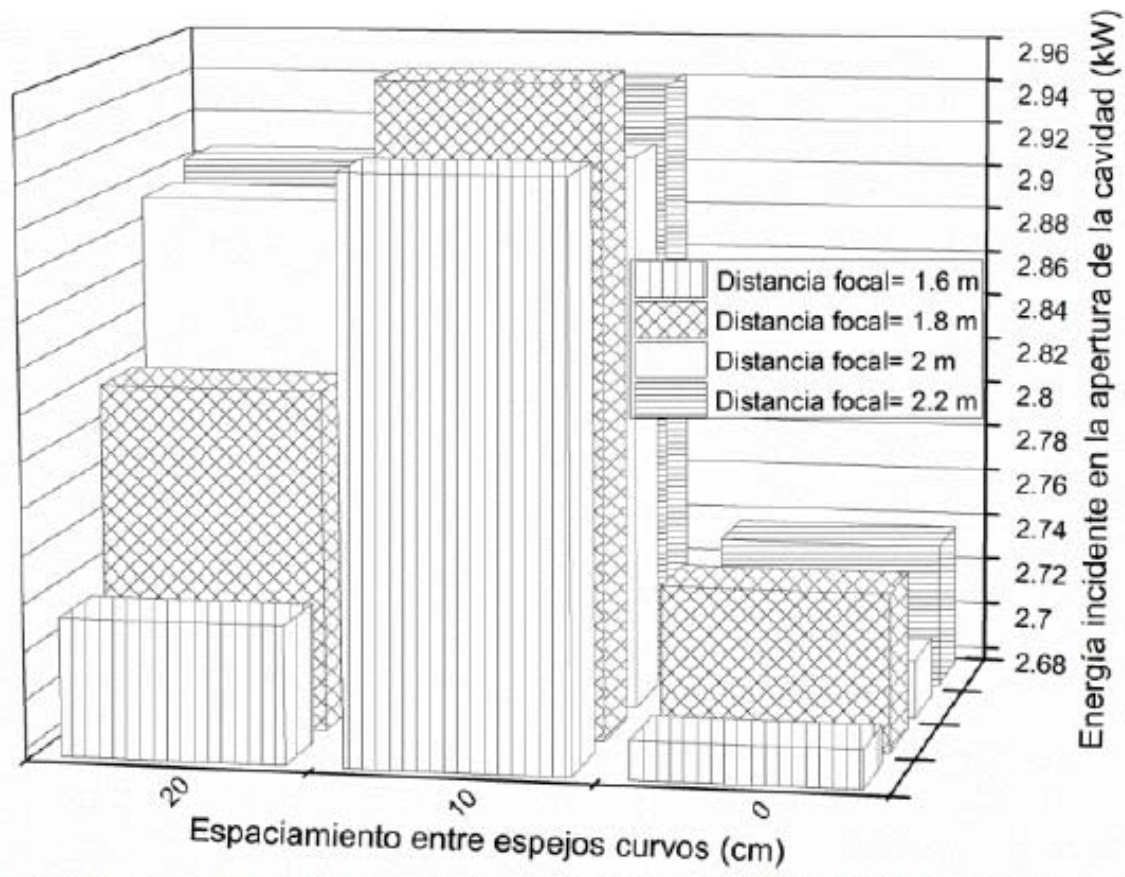

Fig. 13: Captación promedio diaria de las geometrías evaluadas 
También es necesario considerar que aunque el CLF no fue diseñado para el suministro de calefacción en los meses de invierno, este podría ser utilizado a costa de una eficiencia menor ocasionada por las pérdidas de energía en los extremos, las cuales pudiesen haberse incrementado si se hubiese considerado la distancia focal a $2.2 \mathrm{~m}$. Dichas ventajas junto con los resultados previamente obtenidos nos han llevado a especificar las características geométricas del Concentrador Lineal Fresnel como se describe en la Tabla 4.

Tabla 4: Características geométricas del CLF

\begin{tabular}{|l|l|}
\hline Distancia focal $(f)$ & $1.8 \mathrm{~m}$ \\
\hline Ancho total del CLF $\left(W_{C L F}\right)$ & $2.98 \mathrm{~m}$ \\
\hline Número de espejos $(N)$ & 7 \\
\hline Espaciamiento entre espejo $\left(S_{n}\right)$ & $10 \mathrm{~cm}$ \\
\hline Ancho de espejos $(w)$ & $34.30 \mathrm{~cm}$ \\
\hline Profundidad de los espejos $(p)$ & $0.40 \mathrm{~cm}$ \\
\hline Ángulo medio de aceptancia de la cavidad $\left(\theta_{a}\right)$ & $41.67^{\circ}$ \\
\hline Angulo de apertura de los espejos $\left(\phi_{r}\right)$ & $5.50^{\circ}$ \\
\hline Ancho de la cavidad tipo CPC $\left(W_{c a v}\right)$ & $17.45 \mathrm{~cm}$ \\
\hline
\end{tabular}

En la Fig. 14 se muestra el comportamiento operativo horario del concentrador seleccionado y la eficiencia de captación solar correspondiente. Entre los resultados más importantes se obtuvo que la máxima energía captada (12:00 PM) fue de $3.82 \mathrm{~kW}$ con una pérdida de energía de $0.82 \mathrm{~kW}$, una eficiencia de $82.4 \%$ y una pérdida de energía en los extremos de $17.6 \%$, mientras que en la primera o última hora del día (8:00 AM ó 4:00 PM) la energía captada fue de $1.56 \mathrm{~kW}$ con una pérdida de energía de $0.12 \mathrm{~kW}$ y una eficiencia de $33.6 \%$. La forma como se cuantificaron las pérdidas de energía fue considerando los rayos no interceptados por la cavidad debido a bloqueos entre espejos, sombreado de la cavidad, pérdida energía en los extremos, etc.

En cuanto al diseño de la cavidad tipo CPC, primeramente se consideró un ancho de apertura de $17.45 \mathrm{~cm}$ con base en los resultados obtenidos, sin embargo dicho valor fue modificado al ser ajustado el diámetro de absorbedor por el diámetro comercial inmediato superior, ocasionando un reajuste en la cavidad como sigue: ancho del CPC sin truncar $\left(W_{\text {cav }}\right)$ de $19.73 \mathrm{~cm}$, altura de la cavidad $(\mathrm{H})$ de $17.73 \mathrm{~cm}$, diámetro externo del absorbedor tubular $\left(\mathrm{D}_{\mathrm{a}}\right)$ de $4.21 \mathrm{~cm}$, concentración geométrica de la cavidad $\left(\mathrm{C}_{C P C}\right)$ de 1.50 y concentración geométrica del CLF $\left(\mathrm{C}_{C L F}\right)$ de 11.87 .

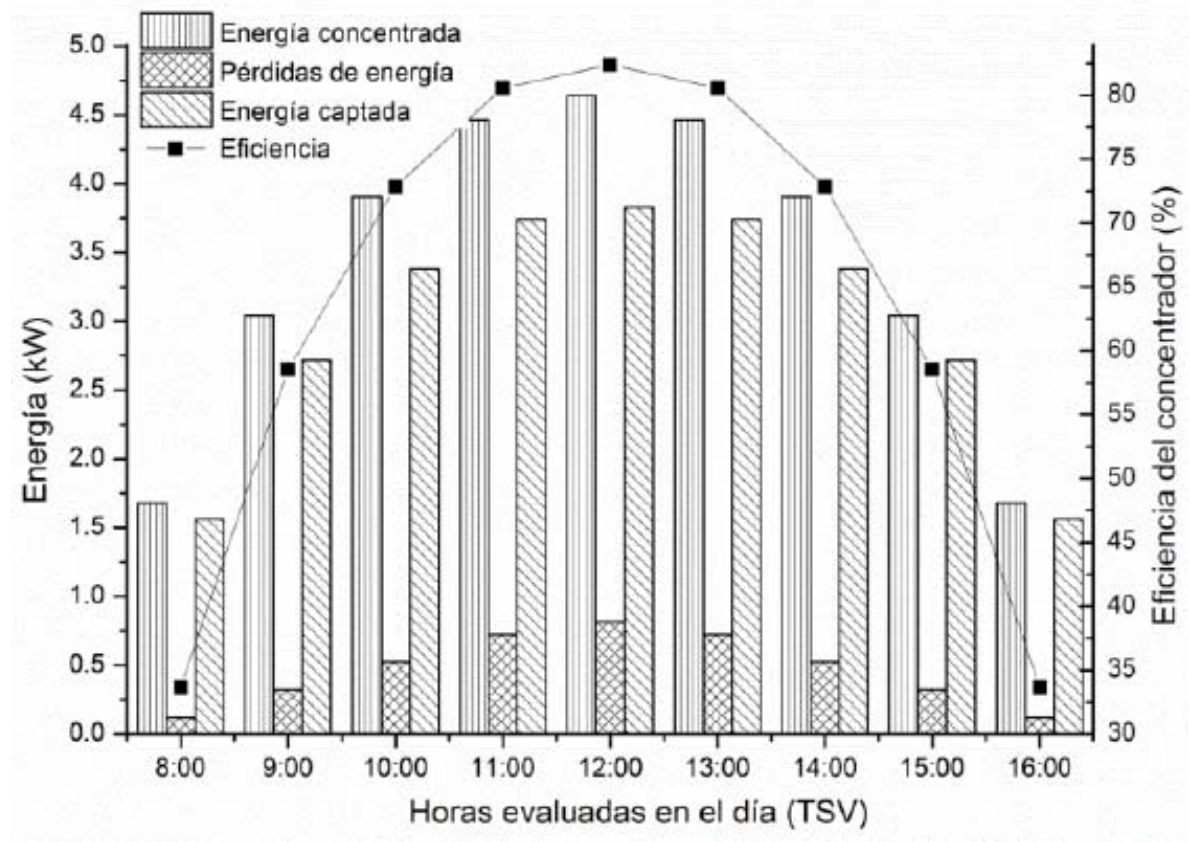

Fig. 14: Comportamiento operativo del CLF seleccionado 
Con base en $W_{\text {cav }}$ de $19.73 \mathrm{~cm}$ y en el diámetro del absorbedor tubular de $4.21 \mathrm{~cm}$, se establece que la geometría tipo CPC permite una reducción en el diámetro del tubo absorbedor de 4.7 veces menos en comparación con el resultante en una geometría de simple tubo absorbedor sin cavidad, lo cual se puede ver reflejado como una reducción en las pérdidas ópticas y térmicas del CLF que ayudan a incrementar la eficiencia global del colector solar.

Finalmente, en la Fig. 15 se muestra una fotografía del CLF construido utilizando la metodología propuesta dentro de este estudio. Con este prototipo se buscará efectuar en futuros trabajos la validación experimental entre el modelo matemático general (análisis geométrico, óptico y térmico) y el comportamiento operativo del concentrador, así como el acoplamiento directo y operabilidad con un sistema de enfriamiento por absorción avanzado solar-GAX.

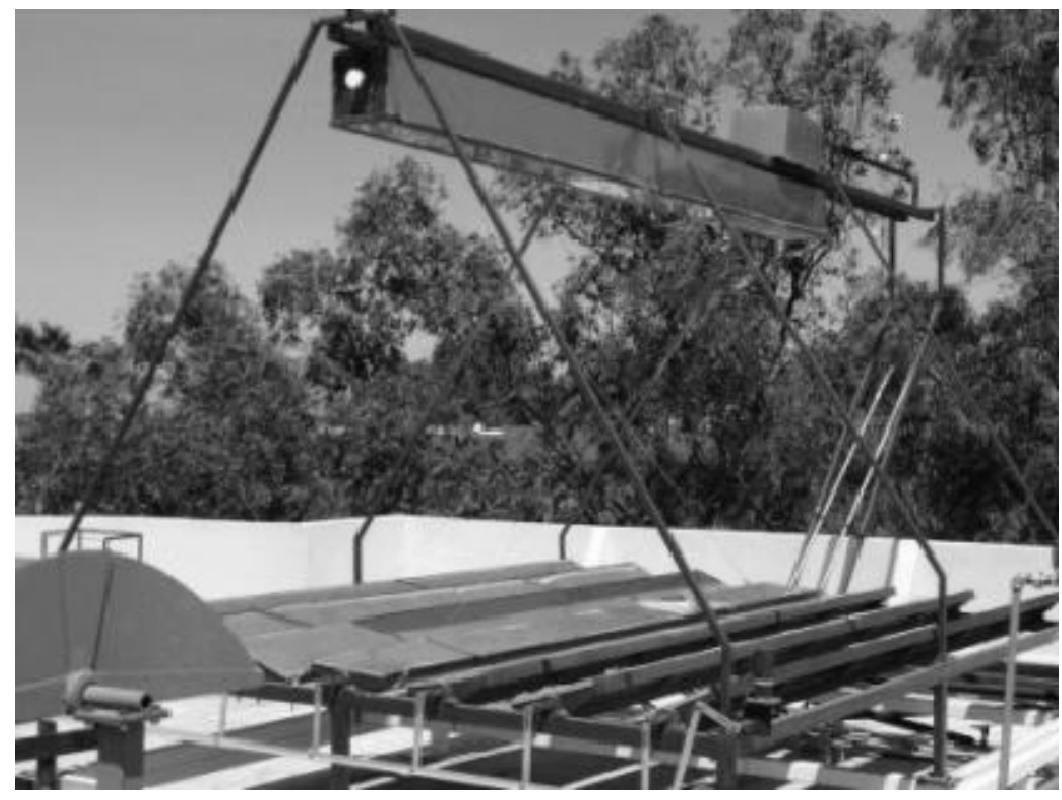

Fig. 15: Prototipo del CLF construido en la plataforma solar del Centro de Estudio de las Energías Renovables del Instituto de Ingeniería

\section{CONCLUSIONES}

De acuerdo a los resultados obtenidos, se concluye que a medida que el espaciamiento entre espejos del CLF se incrementa, la inclinación de estos se ve afectada de una manera importante, de tal forma que se genera un desaprovechamiento en el área reflectiva (campo de reflexión). Aplicando la metodología propuesta, se puede establecer un espaciamiento adecuado que produzca el mínimo sombreado entre espejos y la mínima dispersión de los rayos solares reflejados hacia la cavidad. Entre los beneficios alcanzados por el espaciamiento entre espejos se logró un incremento de la captación solar del 7.37 \% con respecto a la geometría de cero espaciamiento. La máxima energía captada (12:00 PM) fue de $3.82 \mathrm{~kW}$ con una pérdida de energía de $0.82 \mathrm{~kW}$, una eficiencia de $82.4 \%$ y una pérdida de energía en los extremos de $17.6 \%$. El uso de CLF con longitudes significativas o la incorporación de diseños con cavidades desplazables, ayudan a contrarrestar las pérdidas de coseno ocasionadas por el ángulo de incidencia, generando un mayor aprovechamiento de la energía concentrada. La implementación de la geometría tipo CPC permite una reducción en el diámetro del tubo absorbedor de 4.7 veces menos en comparación con el resultante en una geometría de simple tubo absorbedor sin cavidad, lo cual se puede ver reflejado como una reducción en las pérdidas ópticas y térmicas del CLF que ayudan a incrementar la eficiencia global del colector solar. Finalmente, se establece que la herramienta metodológica propuesta para diseñar, analizar y optimizar óptica y geométricamente un concentrador lineal Fresnel, representa una alternativa fácil y eficiente que permite establecer todos los parámetros geométricos relacionados a este tipo de colectores solares.

\section{NOMENCLATURA}
A área, $\mathrm{m}^{2}$
$A^{*} \quad$ distancia formada desde la tangente del absorbedor al inicio de la parábola, $\mathrm{cm}$
C concentración teórica o geométrica 


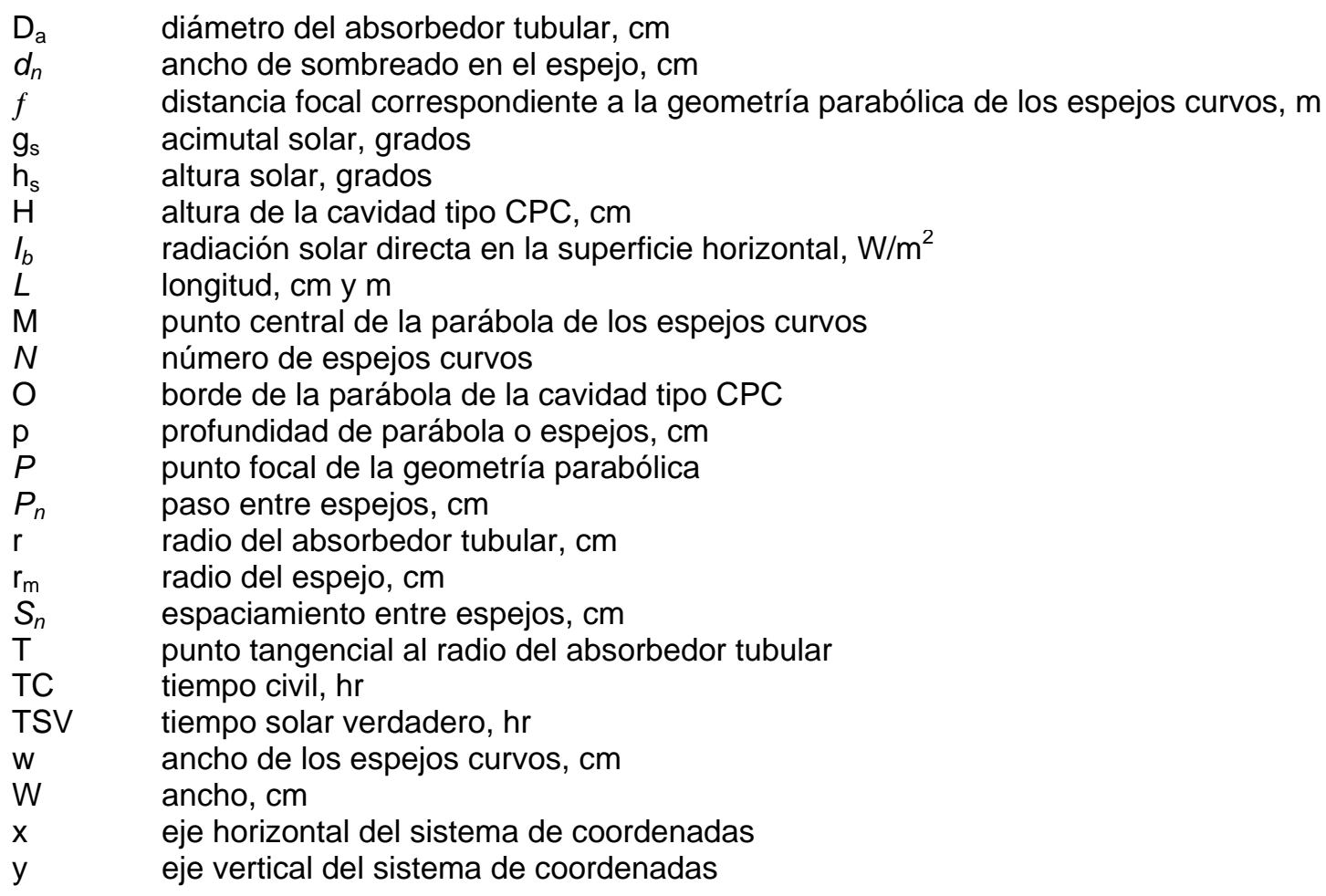

\section{Símbolos griegos}

$\theta \quad$ ángulo de incidencia, grados

$\theta_{a} \quad$ ángulo medio de aceptancia, grados

$\theta_{i} \quad$ ángulo de inclinación de espejos (i=1, 2, 3...7), grados

$\phi \quad$ parámetro angular usado para describir la geometría de la superficie del reflector CPC

$\phi_{r} \quad$ ángulo de apertura, grados

\section{Subíndices}

$\begin{array}{ll}\text { a } & \text { absorbedor } \\ \mathrm{C} & \text { cubierta de vidrio } \\ \text { cav } & \text { cavidad } \\ \text { CLF } & \text { concentrador lineal Fresnel } \\ \text { CPC } & \text { concentrador parabólico compuesto } \\ N & \text { número de espejos curvos } \\ r & \text { reflector CPC }\end{array}$

\section{AGRADECIMIENTOS}

Los autores agradecen el apoyo a CONACYT-Gobierno de Baja California por el apoyo económico a través del proyecto FOMIX 76927.

\section{REFERENCIAS}

Barale, G., A. Heimsath, P. Nitz y A. Toro, Optical Design of a Linear Fresnel Collector for Sicily, Solar Paces, $16^{\text {th }}$ International Symposium on Concentrating Solar Power and Chemical Energy Systems, Perpignan Francia, 21 al 24 de Septiembre (2010).

Duffie J. y W. Beckman, Solar Engineering of Thermal Processes. Segunda edición, 3-45. Wiley Easten Ltd., New York, USA (1991).

Grena R. y P. Tarquini, Solar Linear Fresnel Collector Using Molten Nitrates as Heat Transfer Fluid, Energy: 36(2), 1048-56 (2011). 
Häberle A., M. Berger, F. Luginsland, C. Zahler, M. Baitsch, H. Henning y M. Rommel, Linear Concentrating Fresnel Collector for Process Heat Applications. Solar Paces, 13th International Symposium on Concentrating Solar Power and Chemical Energy Technologies, Sevilla España, 20 al 23 de Junio (2006).

Khonkar H. E. I. y A. A. M. Sayigh, Optimization of the Tubular Absorber Using a Compound Parabolic Concentrator, Renewable Energy: 6(1), 17-21 (1995).

Lentz H. Á., S. R. Almanza, R. S. López y T. R. Cadenas, Available Energy and Cumulative Frequency Curves for Parabolic Trough Concentrators with Alignment North-South, in the Northwest of Mexico. Ingeniería. Investigación y Tecnología, 10(2), 159-166 (2009).

Mathur S. S., B.S. Negi y T. C. Kandpal, Geometrical Designs and Performance Analysis of a Linear Fresnel Reflector Solar Concentrator with a Flat Horizontal Absorber. International Journal of Energy Research: 14(1), 107-124 (1990).

Mathur S. S., T. C. Kandpal y B.S. Negi, Optical Design and Concentration Characteristics of Linear Fresnel Reflector Solar Concentrators-I. Mirror Elements of Varying Width. Energy Convers. Mgmt.: 31(3), 205-219 (1991a).

Mathur S. S., T. C. Kandpal y B.S. Negi, Optical Design and Concentration Characteristics of Linear Fresnel Reflector Solar Concentrators-II. Mirror Elements of Equal Width. Energy Convers. Mgmt.: 31(3), 221-232 (1991b).

Mills D. y G. Morrison, Compact Linear Fresnel Reflector Solar Thermal Powerplants. Solar Energy: 68(3), 263-283 (2000).

Nixon J. D. y P. A. Davies, Cost-Exergy Optimization of Linear Fresnel Reflectors. Solar Energy: 86(1), 147156 (2012).

Singh P., S. Ganesan y G. Yadav, Technical note - Performance Study of a Linear Fresnel Concentrating Solar Device. Renewable Energy: 18(3), 409-416 (1999).

Singh P. L., R. M. Sarviya y J. L. Bhagoria, Heat Loss Study of Trapezoidal Cavity Absorbers for Linear Solar Concentrating Collector. Energy Conversion and Management: 51(2), 329-337 (2010).

Sootha G. D. y B.S. Negi, A Comparative Study of Optical Designs and Solar Flux Concentrating Characteristics of a Linear Fresnel Reflector Solar Concentrator with Tubular Absorber. Solar Energy Materials and Solar Cells: 32(2), 169-186 (1994).

Velázquez N., O. García-Valladares, D. Sauceda y R. Beltran, Numerical Simulation of a Linear Fresnel Reflector Concentrator Used as Direct Generator in a Solar-GAX cycle. Energy Conversion and Management: 51(3), 434-445 (2010). 